\title{
Spectrofluorimetric method for atenolol determination based on gold nanoparticles
}

\author{
ESAM BAKIR ${ }^{1,2 *}$ \\ MOHAMED GOUDA ${ }^{1,3}$ \\ AHMED ALNAJJAR ${ }^{1}$ \\ WALEED E. BORAIE ${ }^{1,2}$ \\ ${ }^{1}$ Department of Chemistry \\ College of Science, King Faisal \\ University, Hofuf, Saudi Arabia \\ ${ }^{2}$ Department of Chemistry \\ Faculty of Science, Ain Shams \\ University, Cairo, Egypt \\ ${ }^{3}$ Textile Research Division \\ National Research Center, Dokki \\ Cairo, Egypt
}

Accepted January 21, 2018

Published online February 19, 2018

\begin{abstract}
A simple and sensitive spectrofluorimetric method for determination of atenolol (ATE) using gold nanoparticles (AuNPs) was developed. The method is based on the quenching effect of atenolol on photoluminescence of AuNPs at $\lambda_{\mathrm{em}}=705 \mathrm{~nm}$. Variables affecting luminescence of gold nanoparticles such as the solvent, $\mathrm{pH}$ value and surfactant were studied and optimized. The method was preliminarily validated according to ICH guidelines. A linear correlation was recorded within the range of $1.0-10 \mu \mathrm{g} \mathrm{mL}^{-1}$ ATE with the coefficient of determination $R^{2}$ of 0.999 . The limit of detection and limit of quantitation for atenolol were found to be 0.87 and $2.64 \mu \mathrm{g} \mathrm{mL}^{-1}$, resp. Good recoveries in the range of 98.7-100.0\% were obtained for spiked samples. The proposed method was applied successfully to assaying atenolol in pharmaceuticals formulations.
\end{abstract}

Keywords: atenolol, spectrofluorimetry, gold nanoparticles

Atenolol, 4-(2-hydroxy-3-isopropylaminopropoxy)phenylacetamide (ATE), is a betablocker that affects the heart and circulation. Atenolol is used to treat angina and hypertension. Atenolol is also used to lower the risk of death after a heart attack (1).

Previous studies applied spectrofluorimetry to estimation of atenolol in aqueous solutions and samples of human urine because of its sensitivity, selectivity and low-cost instrumentation (2). Fluorescence was used to detect atenolol without and with solid-phase extraction (3). Photoluminescence of metal nanoparticles that offer emission of light without requiring conjugation with luminous dyes is the basis for the new method (4).

The photo-physical properties of colloidal gold nanoparticles are unique in showing stable and non-bleaching fluorescence, which was already widely used in a variety of molecularly targeted biological applications, including multiphoton and correlative microscopy (5). In this study, novel, simple, sensitive and selective gold nanoparticles (AuNPs) were developed for determination of ATE.

\footnotetext{
*Correspondence; ebakir@kfu.edu.sa; esambakir99@yahoo.com
} 


\section{EXPERIMENTAL}

\section{Materials}

Atenolol, gold(III) chloride trihydrate $\left(\mathrm{HAuCl}_{4}\right)$, cetyl-trimethyl-ammonium bromide (CTAB), sodium dodecyl sulfate (SDS) surfactant and HPLC grade solvents such as methanol, acetonitrile (ACN) and dimethylsulphoxide (DMSO) were purchased from Sigma Aldrich (USA). A commercial pharmaceutical formulation (Normoten-100, Jazeera Pharmaceutical Industries, Saudi Arabia) was used.

\section{Synthesis and physical characterization of gold nanoparticles}

Gold nanoparticles were synthesized by the citrate reduction method according to the reported method (6).

Absorption and photo-luminescence spectra were measured on spectrophotometer and spectrofluorimeter models USB4000 and USB4000FL, resp. (Ocean Optics Devices, USA). Diameter and colloidal properties of gold nanoparticles were estimated using scanning electron microscopy (SEM) on a Phillips CM201C microscope at $80 \mathrm{kV}$ (Phillips, The Netherlands).

\section{ATE standard solutions and calibration}

An accurately weighed amount of ATE was dissolved in methanol to get a stock solution of $50 \mu \mathrm{g} \mathrm{mL}-1$. Working ATE solutions in the concentration range of $1-10 \mu \mathrm{g} \mathrm{mL} \mathrm{L}^{-1}$ were transferred into a series of $10-\mathrm{mL}$ calibrated flasks containing $300 \mu \mathrm{L}$ of $0.277 \mathrm{mmol} \mathrm{L}^{-1}$ colloidal AuNPs.

\section{Procedure for commercial tablets}

Fine powder of Normoten-100 tablets (equivalent to $100 \mathrm{mg}$ of atenolol) was accurately weighed and placed in a $100-\mathrm{mL}$ flask containing methanol. The solution was sonicated, filtered and then made up with methanol to $10 \mu \mathrm{g} \mathrm{mL} \mathrm{L}^{-1}$ of atenolol.

\section{Quenching of photoluminescent AuNPs by ATE}

The Stern-Volmer relationship was explored for the kinetics of photo-physical intermolecular deactivation of photoluminescent AuNPs by atenolol according to:

$$
\frac{I_{0}}{I}-1=K_{\mathrm{SV}}[Q]
$$

where $I_{0}$ and $I$ are luminescence intensity of AuNPs in the absence and in the presence of different atenolol concentrations at $\lambda_{\mathrm{em}}=705 \mathrm{~nm}$, [Q] is the concentration of the quencher and $K_{\mathrm{SV}}$ is the Stern-Volmer constant as a measure of sensor sensitivity (7).

\section{Method validation}

Validation of the method was done according to the ICH Q2 (R1) guideline (8).

Linearity and range. - Linearity was established from the calibration curve of photoluminescence of AuNPs $v$ s. atenolol concentration estimating $R^{2}$. Limits of detection and 
quantitation ( $L O D$ and $L O Q$, resp.) for the proposed method were evaluated as per ICH guidelines using the formulae:

$$
L O D=3.3 \sigma / S \text { and } L O Q=10 \sigma / S
$$

where $\sigma$ is standard deviation of the response (standard deviation of y-intercepts of the regression line), $S$ is the slope of the calibration line.

Precision and accuracy. - Intra-day and inter-day precision were investigated by evaluating 3 sample replicates on the same day and on 3 different days of a week. Results are expressed as RSD.

Accuracy was established as the percent of recovery of ATE for three standard additions to the sample. Three different concentrations were added to a fixed amount of the prepared drug sample, standard Normoten, and the total was analyzed by the studied method. Each analysis was carried out three times.

Specificity. - Specificity of the method for atenolol was evaluated in the presence of interfering substances such as excipients, degradants and matrix components (starch, lactose, glucose and sodium chloride). Excipient concentrations were 5, 10 and $20 \mu \mathrm{g} \mathrm{mL}$ mixed with a fixed concentration of atenolol $\left(5 \mu \mathrm{g} \mathrm{mL} \mathrm{L}^{-1}\right)$.

Robustness. - For the determination of method robustness, three factors were examined: reaction time, change of temperature and change of the order of reagent addition.

\section{RESULTS AND DISCUSSION}

\section{Diameter of $A$ uNPS}

AuNPs appeared as small dark spherical dots. Fig. 1 shows the SEM images of AuNPs, which reveal that the average size of AuNPs with the shape of nanoclusters was $22.8 \pm 8.0 \mathrm{~nm}$.

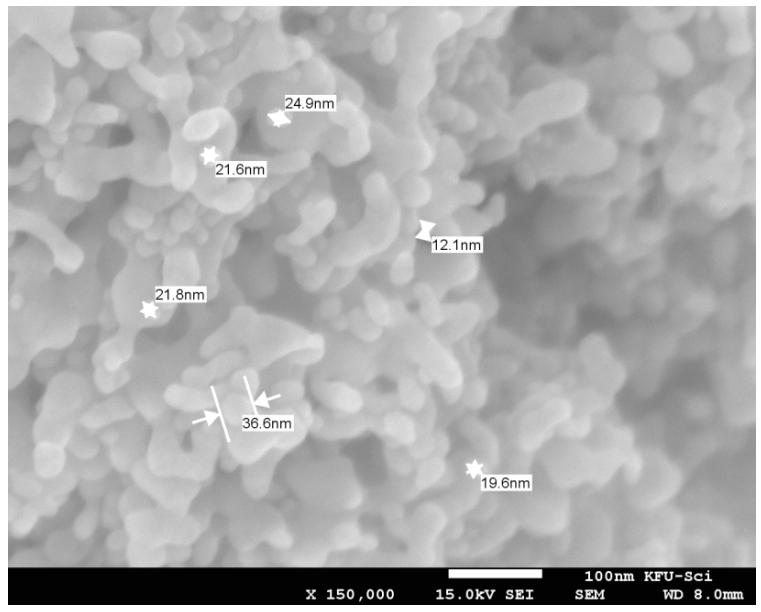

Fig. 1. SEM image of AuNPs prepared by the citrate reduction method in DMSO. 


\section{Factors affecting AuNP luminescence}

Solvent effect. - The absorption and luminescence spectra are shown in Fig 2. Absorbance of Au nanoparticles at $525 \mathrm{~nm}$ decreased whenever the dipole moment of the solvent decreased. The gold nanoparticles aggregated and flocculated at room temperature in a nonpolar solvent such as cyclohexane while the strength of solvent polarity maintained the AuNP particles in suspension form.

In the case of protic solvents, the absorbance of AuNPs is slightly red-shifted as the polarity decreases. This might be attributed to the electrostatic interaction of the solvent $\mathrm{OH}$ group with AuNP citrate capping. In the case of acetonitrile, the absorbance is also red-shifted. It is worth noting that the peak in this case is shown to be broad. In the case of CAN, the AuNPs appear to be aggregated, since ACN has low affinity towards AuNPs (9). In the case of DMSO, the sulfuric group can envelope AuNPs as a further protection layer, resulting in the lowest quenching of AuNP luminescence (10).

Effect of $\mathrm{pH}$. - The absorbance and photoluminescence of colloidal AuNP dispersions was measured at different $\mathrm{pH}$ values (4-10). At $\mathrm{pH} \leq 4$, the negative charge of citrate anions surrounding the gold nanoparticles became fully protonated and the Au nanoparticles were aggregated; hence the stability of the suspension form decreased. At $\mathrm{pH} \geq 10.0$, the gold nanoparticle surface was fully deprotonated and their stability was improved (11).

Effect of $\mathrm{KCl}$ solution. - The absorbance and photoluminescence properties of AuNPs were measured at $635 \mathrm{~nm}$ using different concentrations of $\mathrm{KCl}\left(0.1-1.00 \mathrm{mmol} \mathrm{L}^{-1}\right)$. At low ionic strength, small aggregates were formed of gold nanoparticles, which were stable over a long period of time. We found that the AuNPs showed faster aggregation and precipitation at $0.6 \mathrm{mmol} \mathrm{L}^{-1} \mathrm{KCl}(12)$.

Effect of surfactant. - The absorption band of gold nanoparticles was affected by different concentrations of CTAB (10-50 $\left.\mathrm{mmol} \mathrm{L}^{-1}\right)$ and SDS (10-50 $\left.\mathrm{mmol} \mathrm{L}^{-1}\right)$. The AuNPs aggregated at $10 \mathrm{mmol} \mathrm{L}^{-1}$ of cationic CTAB micelles due to the strong interaction of the cationic head group

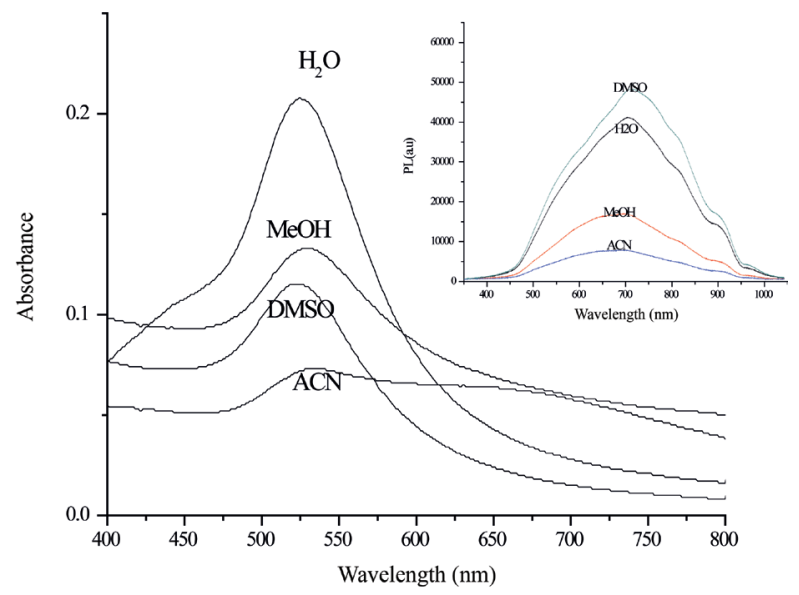

Fig. 2. Absorption and photoluminescence spectra of AuNPs using different solvents. 
of CTAB micelles with citrate capping of AuNPs. At higher concentrations $\left(>10 \mathrm{mmol} \mathrm{L}^{-1}\right)$, the AuNPs became more stable because the CTAB micelles formed a bilayer over citrate capped AuNPs. In the case of $10 \mathrm{mmol} \mathrm{L}^{-1}$ of anionic SDS, the AuNPs did not aggregate due to the electrostatic repulsion between the negative head group $\left(-\mathrm{OSO}_{3}{ }^{-}\right)$and citrate capping on AuNPs. AuNPs were stabilized at $>10 \mathrm{mmol} \mathrm{L}^{-1}$ of SDS micelles since the SDS micelles adsorbed on the surface of citrate-capped AuNPs (13).

\section{Analytical validation}

Linearity. - The calibration line was established in the range of $0.1-10 \mu \mathrm{g} \mathrm{mL}^{-1} \mathrm{ATE}$, $R^{2}=0.9998$. Table I shows the $L O D$ and $L O Q$ values of 0.87 and $2.64 \mu \mathrm{g} \mathrm{mL} L^{-1}$ ATE, resp.

Sensitivity and stability. - The high value of molar absorptivity $(\varepsilon)$ and low values of $L O D / L O Q$ indicate high sensitivity of the proposed method, as shown from Table I. The luminescence intensity of AuNPs was stable for $24 \mathrm{~h}$. Using the spike standard, namely, after adding atenolol, recovery of luminescence of AuNPs-ATE was 99.5 $\pm 0.0_{1} \%$ (mean $\pm \mathrm{SD}$, $n=3$ ) and the RSD value was less than $1 \%$. The luminescence of AuNPs-ATE was measured at time intervals of 1,10 and $60 \mathrm{~min}$ and the temperature intervals were 20,30 and $40{ }^{\circ} \mathrm{C}$.

Accuracy and precision. - Table II shows the accuracy and precision of the proposed method. RSD was $\leq 2.0 \%$ (intra-day) and $\leq 0.4 \%$ (inter-day). In addition, the accuracy of the proposed method was measured by calculating the percent relative error, which varied

Table I. Sensitivity and regression parameters for atenolol by AuNPs as a photo probe

\begin{tabular}{lc}
\hline Analyte & Atenolol \\
\hline Wavelength $(\mathrm{nm})$ & $705 \mathrm{~nm}$ \\
Beer's law limits $(\mu \mathrm{g} \mathrm{mL}-1)$ & $1-10$ \\
Regression equation & $y=a+b x(a=0.0346, b=1.0012)$ \\
Coefficient of determination $\left(R^{2}\right)$ & 0.999 \\
$L O D\left(\mu g \mathrm{~mL}^{-1}\right)$ & 0.87 \\
$L O Q\left(\mu \mathrm{gL}^{-1}\right)$ & 2.64 \\
Molar absorptivity $\left(\varepsilon, \mathrm{L} \mathrm{moL}^{-1} \mathrm{~cm}^{-1}\right)$ & $3.7 \times 10^{2}$ \\
RSD $(\%)$ & 1.2 \\
\hline
\end{tabular}

Table II. Intra-day and inter-day accuracy and precision

\begin{tabular}{ccccccc}
\hline \multirow{2}{*}{$\begin{array}{c}\text { Atenolol } \\
\text { taken } \\
\left(\mu \mathrm{g} \mathrm{mL}^{-1}\right)\end{array}$} & \begin{tabular}{c} 
Intra-day accuracy and precision \\
\cline { 2 - 7 } \\
\cline { 2 - 7 } \\
$\left(\mu \mathrm{g} \mathrm{mL}^{-1}\right)$
\end{tabular} & RSD $(\%)$ & Accuracy* & $\begin{array}{c}\text { ATter-day accuracy and precision } \\
\text { average } \\
\left(\mu \mathrm{mL}^{-1}\right)\end{array}$ & RSD $(\%)$ & Accuracy* \\
\hline 0.100 & 0.102 & 2.0 & $102.0 \pm 1.0$ & 0.103 & 0.3 & $101.0 \pm 0.4$ \\
2.700 & 2.690 & 0.4 & $99.6 \pm 1.0$ & 2.720 & 0.4 & $101.1 \pm 0.4$ \\
7.000 & 7.010 & 0.3 & $100.1 \pm 1.0$ & 7.020 & 0.1 & $100.1 \pm 0.4$ \\
\hline
\end{tabular}

ATE - atenolol

${ }^{*}$ Recovery (\%); mean $\pm \mathrm{SD}, n=3$. 
between 0.1 and $2.5 \%$, and the recovery was $99.6-102.0 \%$ according to the standard addition method. Results presented in Table II reveal a slight effect on the accuracy of the method in various excipients.

Robustness. - The average value of ATE recovery was $99.5 \pm 0.0_{1} \%$ using standard additions (mean $\pm \mathrm{SD}, n=3$ ) and the RSD value was less than $1 \%$. This means that the proposed method is robust with small variations in temperature $\left(20,30\right.$ and $\left.40{ }^{\circ} \mathrm{C}\right)$ and time intervals (1, 10 and $60 \mathrm{~min})$.

Specificity. - Recovery of atenolol was found in the range $99.0-101.0 \%$ with RSD $<2 \%$ using the standard addition method.

\section{Analysis of the commercial formulation}

The proposed method was successfully applied to determination of ATE in the Normoten-100 formulation. The results are given in Table III.

Table IV shows the data obtained by the proposed method compared to reference methods $(14,15)$. Recovery of ATE in Normoten $(100 \mathrm{mg}$ ATE) by the proposed method was

Table III. Determination of atenolol in the pharmaceutical formulation using AuNPs as a photo probe

\begin{tabular}{ccccc}
\hline Formulation & $\begin{array}{c}\text { Normoten } \\
\left(\mu \mathrm{g} \mathrm{mL}^{-1}\right)\end{array}$ & $\begin{array}{c}\text { ATE added } \\
\left(\mu \mathrm{g} \mathrm{mL}^{-1}\right)\end{array}$ & $\begin{array}{c}\text { Total ATE found } \\
\left.(\mu \mathrm{g} \mathrm{mL})^{-1}\right)\end{array}$ & $\begin{array}{c}\text { ATE recovered } \\
(\%)^{\mathrm{a}}\end{array}$ \\
\cline { 2 - 5 } & 3.00 & 0.00 & 2.99 & $99.7 \pm 0.1$ \\
Normoten-100 & 3.00 & 0.50 & 3.51 & $98.0 \pm 2.0$ \\
& 3.00 & 3.00 & 5.99 & $99.7 \pm 0.3$ \\
& 3.00 & 5.00 & 7.99 & $99.8 \pm 0.2$ \\
\hline
\end{tabular}

${ }^{\mathrm{a}}$ Mean $\pm \mathrm{SD}, n=3$.

Table IV. Analysis of atenolol in tablets by the proposed and reference methods

\begin{tabular}{lcccc}
\hline Method & $\begin{array}{c}\text { Label claim } \\
-100 \mathrm{mg} \text { ATE }\end{array}$ & $\begin{array}{c}\text { Reference } \\
\text { method } \\
(\text { recovery, \%) }\end{array}$ & $\begin{array}{c}\text { Linearity } \\
\text { range/LOD } \\
\left(\mu \mathrm{g} \mathrm{mL} \mathrm{mL}^{-1}\right)\end{array}$ & Remarks \\
\hline $\begin{array}{l}\text { Oxidation of drug using } \\
\text { alkaline } \mathrm{KMnO}_{4}(13)\end{array}$ & Blokium & $100.0 \pm 0.5$ & $\begin{array}{c}2.0-14.0 / \\
0.081\end{array}$ & $\begin{array}{c}\text { Less sensitive under } \\
\text { heating }\end{array}$ \\
$\begin{array}{l}\text { Reaction with Phenol Red } \\
\text { in acidic medium (14) }\end{array}$ & Normoten & $98.9 \pm 0.3$ & $\begin{array}{c}0.5-30 / \\
0.038\end{array}$ & No interferences \\
$\begin{array}{l}\text { Quenching of luminescence } \\
\text { of AuNPs by atenolol } \\
\text { (proposed method) }\end{array}$ & Normoten & $100.2 \pm 0.3$ & $\begin{array}{c}2.6-10 / \\
0.870\end{array}$ & $\begin{array}{c}\text { Simple, no heating, short } \\
\text { response time, stable } \\
\text { luminescent reagent }\end{array}$ \\
\hline
\end{tabular}

${ }^{\mathrm{a}}$ Recovery $\pm \mathrm{RSD}, n=3$. 
$100.2 \pm 0.3 \%$ (mean $\pm \mathrm{SD}, n=3$ ). The advantages of this method compared to reference methods was that the proposed method had a short response time, $\sim 5 \mathrm{~s}$, and that the AuNP luminescence was stable, $\sim 60 \mathrm{~min}$.

\section{CONCLUSIONS}

A simple, sensitive, accurate and precise spectrofluorimetric method using AuNPs as a photo probe is suggested for determination of atenolol. The luminescence intensity of AuNPs in different solvents, at different $\mathrm{pH}$ values, at different $\mathrm{KCl}$ concentrations and in the presence of two types of surfactants was studied to obtain optimum conditions. The proposed method can be used for routine analysis of atenolol in pharmaceutical dosage forms.

Acknowledgments. - The authors are grateful to the Dean of Scientific Research at King Faisal University for financial support for project no. 140255.

\section{REFERENCES}

1. J. G. Hardman, L. E. Limbird and A. G. Gilman, Goodman and Gilman's The Pharmacological Basis of Therapeutics, $10^{\text {th }}$ ed., McGraw-Hill, New York, 2001, 1011-1021.

2. F. Belal, S. Al-Shaboury and A. Al-Tamrah, Spectrofluorometric determination of labetolol in pharmaceutical preparations and spiked human urine through the formation of coumarin derivative, J. Pharm. Biomed. Anal. 30 (2002) 1191-1196; https://doi.org/10.1016/s0731-7085(02)00471-5

3. H. Basan and S. Yarımkaya, A novel solid-phase extraction-spectrofluorimetric method for the direct determination of atenolol in human urine, Luminescence 29 (2013) 225-229; https://doi. org/10.1002/bio.2532

4. A. Bavili-Tabrizi, F. Bahrami and H. Badrouj, A very simple and sensitive spectrofluorimetric method based on the oxidation with cerium(IV) for the determination of four different drugs in their pharmaceutical formulations, Pharm. Sci. 23 (2017) 50-58; https://doi.org/10.15171/ps.2017.08

5. Y. Jiang, N. N. Horimoto, K. Imura, H. Okamoto, K. Matsui and R. Shigemoto, Bioimaging with two-photon-induced luminescence from triangular nanoplates and nanoparticle aggregates of gold, Adv. Mat. 21 (2009) 2309-2313; https://doi.org/10.1002/adma.200802312

6. A. D. McFarland, C. L. Haynes, C. A. Mirkin, R. P. V. Duyne and H. A. Godwin, Color my nanoworld, J. Chem. Ed. 81 (2004) 544A-544B; https://doi.org/10.1021/ed081p544a

7. O. Stern and M. Volmer, Decay of fluorescence, J. Röntgen Soc. 15 (1919) 133-133; https://doi. org/10.1259/1rs.1919.0087

8. International Conference on Harmonisation of Technical Requirements for Registration of Pharmaceuticals for Human Use, ICH Harmonised Tripartite Guideline, Validation of Analytical Procedures: Text and Methodology Q2(R1), Current Step 4 version, November 2005; https://www.ich.org/fileadmin/Public_Web_Site/ICH_Products/Guidelines/Quality/Q2_R1/Step4 /Q2_R1_Guideline.pdf; last access date Dec 11, 2017.

9. K. G. Thomas, B. I. Ipe and P. K. Sudeep, Photochemistry of chromophore-functionalized gold nanoparticles, Pure Appl. Chem. 74 (2002) 1731-1738; https:// doi.org/10.1351/pac200274091731

10. V. Amendola, G. A. Rizzi, S. Polizzi and M. Meneghetti, Synthesis of gold nanoparticles by laser ablation in toluene: quenching and recovery of the surface plasmon absorption, J. Phys. Chem. B 109 (2005) 23125-23128; https://doi.org/10.1021/jp055783v 
11. K. Wagers, T. Chui and S. Adem, Effect of $\mathrm{pH}$ on the stability of gold nanoparticles and their application for melamine detection in infant formula, IOSR J. Appl. Chem. 7 (2014) 15-20; https://doi. org/10.9790/5736-07821520

12. R. Pamies, J. G. H. Cifre, V. F. Espín, M. Collado-González, F. G. D. Baños and J. G. de la Torre, Aggregation behaviour of gold nanoparticles in saline aqueous media, J. Nanopart. Res. 16 (2014) Article ID 2376 (11 pages); https://doi.org/10.1007/s11051-014-2376-4

13. J. Lim, N.-E. Lee, E. Lee and S. Yoon, Surface modification of citrate-capped gold nanoparticles using CTAB micelles, Bull. Kor. Chem. Soc. 35 (2014) 2567-2569; https://doi.org 10.5012/ bkcs.2014.35.8.25674

14. A. M. El-Didamony and M. A. Moustafa, Direct spectrophotometric determination of atenolol and timolol anti-hypertensive drugs, Int. J. Pharm. Pharm. Sci. 9 (2017) 47-53; https://doi.org/10.22159/ ijpps.2017v9i3.16198

15. I. E. H. Elgailani and T. H. Alghamdi, Development of spectrophotometric method for the determination of atenolol in Normoten drug, Int. J. Chem. 9 (2017) 58-64; https://doi.org/0.5539/ijc. v9n1p58 